\title{
EVALUACIÓN ERGONÓMICA EN EL MÓDULO DE PRÉSTAMOS DE UNA BIBLIOTECA DE UNIVERSIDAD PÚBLICA
}

\author{
ERGONOMIC EVALUATION IN THE LOAN MODULE OF A PUBLIC \\ UNIVERSITY LIBRARY
}

\begin{abstract}
Alejandra García Becerra1", Julio Cesar Cano Gutiérrez, Elsie Vanessa Ensaldo Rentería', Claudia Camargo Wilson', Jesús Everardo Olguín Tiznado ${ }^{1}$, Juan Andrés López Barreras ${ }^{1}$
\end{abstract}

\section{RESUMO}

El trabajo en oficina implica mantener posturas fijas por periodos largos, lo que conlleva al desarrollo de Trastornos Músculo Esqueléticos, es necesario una evaluación ergonómica para evitar problemas de salud. El objetivo es evaluar por medio de métodos ergonómicos el riesgo de carga postural de los trabajadores de área de préstamo de una biblioteca, para disminuir los problemas de salud asociados a trastornos musculo esqueléticos del área administrativa; Se evaluó una mujer y un hombre y se analizaron tres ciclos de trabajo: préstamo, devolución y multa. Las evaluaciones fueron con el método Rapid Office Strain Assessment (ROSA) y (Rapid Entire Body Assesment (REBA) respectivamente, se tomaron medidas tanto antropométricas como del área de trabajo, y se implementaron mejoras. Los resultados obtenidos a través de los métodos permitieron indagar sobre la necesidad de realizar acciones de mejora para atender las posturas inadecuadas y la organización del espacio de trabajo. Una vez implementadas las recomendaciones se obtienen resultados favorables, mejora la postura de los trabajadores, disminuye el nivel de riesgo de REBA de 9 a 3 para el caso de la mujer y de 6 a 2 para el caso del hombre, concluyendo que las recomendaciones fueron favorables para evitar o disminuir los Trastornos Músculo Esqueléticos.

Palabras clave: Trabajo estático, carga postural, trastorno musculo esqueléticos, desordenes del trauma acumulado, evaluación ergonómica, oficina. 


\section{ABSTRACT}

Office work involves maintaining fixed postures for long periods, which leads to the development of skeletal muscle disorders, which is why an ergonomic evaluation is necessary to avoid health problems. The objective of this study is to evaluate, by means of ergonomic methods, the risk of postural loading of workers in the loan area of a library, in order to reduce the health problems associated with musculoskeletal disorders in the administrative area; A woman and a man were evaluated and three work cycles were analyzed: loan, refund and fine. The evaluations were with the Rapid Office Strain Assessment (ROSA) y (Rapid Entire Body Assesment (REBA) method respectively, both anthropometric and work area measurements were taken, and improvements. The results obtained through the methods allowed to inquire about the need to carry out improvement actions to address the inadequate postures and the organization of the workspace. Once the recommendations are implemented, favorable results are obtained, the workers' position improves, the risk level of REBA decreases from 9 to 3 for the case of the woman and from 6 to 2 for the case of the man, concluding that the recommendations were favorable to avoid or diminish the Skeletal Muscle Disorders

Keywords: Static work, postural load, skeletal muscle disorder, accumulated trauma disorders, ergonomic evaluation, office.

\section{INTRODUCCION}

El trabajo en el área de oficina se encuentra en constante crecimiento y con ello una alta prevalencia de desórdenes musculo esqueléticos relacionados con este tipo de labor, entre el 40-80\%. (Robertson et al., 2013). Entre los años 1830-1850, se comenzaron a realizar estudios de productividad en el área laboral, correlacionándose las molestias con el tipo de trabajo (DeRango et al., 2003). Desde entonces los problemas de salud causados por trabajo, tienen como consecuencia perdidas millonarias en el área de productividad, sanitaria y social. Por lo cual muchos estudios están dirigidos a identificar la naturaleza del tipo de lesiones (Fonseca \& Moraga 2010).

La carga de trabajo estática junto con factores psíquicos, orgánicos y un entorno incómodo de trabajo, conlleva a una fatiga muscular (Minsal 2012). El trabajo administrativo requiere mantener la misma postura por largos periodos de tiempo lo que puede tener efectos nocivos para la salud (Bogaert et al., 2016). Mantener una postura estática dentro del lugar de trabajo, contribuye a problemas relacionados con el dolor de cuello, hombro, espalda superior y escapular, cabeza, fatiga visual, dolor de brazo radicular; disfunción del antebrazo, la muñeca y el dedo; entumecimiento y hormigueo en las manos; dolor lumbar; y síntomas de pierna radicular (Nava et al., 2013) Los problemas de salud proporcionales a horas semanales sentados frente a la computadora conllevan al desarrollo de desórdenes musculo esqueléticos (Muñoz \& Vanegas, 2012). Los Trastornos Musculo Esqueléticos (TME) son lesiones de músculos, tendones, nervios y articulaciones, sus síntomas predominantes son el dolor, inflamación y dificultad para realizar algunos movimientos (Rosario y Amezquita, 
2014) representan un fuerte impacto socioeconómico debido a su alta prevalencia con consecuencias debido a su alta prevalencia. (López Torres et al., 2014)(Pouliakas Theodossiou, 2013).

Actualmente existe una gran cantidad de métodos ergonómicos que se enfocan en medir el nivel de riesgo. Una evaluación ergonómica en el área laboral incita a la implementación de un cambio (Hignett y McAtamney, 2000). Algunos de estos métodos son específicos en evaluar el riesgo de un trastorno musculo esqueléticos, los que fueron desarrollados por investigadores para áreas específicas en ciertas situaciones. (Diego-Mas et al., 2015). Generalmente estos métodos no resuelven el problema ergonómico, si no que recopilan datos los cuales no tienen precisión, proporcionando resultados amplios, suelen ser sencillos de usar, y se aplican a una gama de situaciones de trabajo. Estas técnicas parecen adaptarse mejor a las necesidades de los profesionales de la seguridad y la salud en el trabajo que tienen a su disposición recursos y tiempo limitados y necesitan una base para establecer prioridades de intervención (Genaidy et al.,1994)

El método ROSA (Rapid Office Strain Assessment) ha sido considerado válido en la evaluación de riesgos de oficina. Este método ergonómico identifica características únicas del trabajo en oficina, y genera recomendaciones para optimizar el diseño entre los muebles y el trabajador (Sonne et al., 2012). Siendo esta una herramienta de cribado basada en la orientación de imagen para la cuantificación de una exposición de factores de riesgo para los trabajadores generando una evaluación rápida y sistemática de los riesgos posturales al trabajador (Liebregts, et. al.,2016). El análisis puede llevarse a cabo antes y después de una intervención para demostrar que la intervención ha trabajado para reducir el riesgo de lesiones (Prieto et. al., 2018). Matos y Arezes (2015) trabajando con el método ROSA han resaltado el mal uso del mobiliario, cuando los trabajadores no utilizan el descansabrazos genera un aumento de tención en el cuello, y hombros.

El método REBA (Rapid Entire Body Assessment), realiza análisis postural con sensibilidad a posturas de trabajo impredecibles (Hignett y McAtamney 2000). Este método fue diseñado para evaluar el riesgo asociado con la postura del trabajador y la aparición de molestias musculo esqueléticas (Lasota 2014).

Wineman (2015) menciona la importancia de considerar las medidas antropométricas de los trabajadores de oficina, los ángulos y las longitudes que alcanzan las extremidades en una posición, para el confort y disminución de la fatiga. (Rebelo et al., 2000) trabajando sobre evaluaciones ergonómicas en sillas, han reportado que la altura y profundidad de las sillas no fue la adecuada para un $40 \%$ de los participantes.

El mobiliario, malas posturas adoptadas y la falta de información ergonómica de los trabajadores del área de préstamos en la biblioteca universitaria, se supone está relacionado con los problemas y dolencias musculares de los trabajadores (Minsal, 2012). Este tipo de trabajo exige mantener posiciones forzadas, sostenidas en el área laboral que estan correlacionadas con problemas de salud. Existe una relación epistemológica y estadística entre ambas variables (Natarén et al., 2004)

El método REBA ha sido reportado como una herramienta ergonómica de utilidad para la identificación de sobrecarga postural, en trabajadores administrativos (López Torres et al., 2014; Munoz \& Vanegas, 2012; (Nava et al., 2013). 
Diversos estudios han señalado que el trabajo sedentario tiene consecuencias graves en la salud, incluso puede afectar las labores cotidianas diarias, estas consecuencias van desde la obesidad hasta TME. Cuando se excede la capacidad de respuesta bilógica del individuo respecto a los requerimientos físicos de postura fuerza y movimiento, y no existe una adecuada respuesta biológica se llegan a presentar lesiones musculo esqueléticas (Castillo et al., 2009). EL mantener una misma postura por tiempos excesivos, en posturas inadecuadas incluyendo movimientos y fuerzas para las cuales no está preparado propician la fatiga (Arbeláez et al., 2011).

Una de las áreas más relevantes de una universidad es la biblioteca, y parte de las actividades que realiza el personal administrativo de la biblioteca consiste en el préstamo, devolución y pago de multas, lo cual genera que los trabajadores adopten posturas inadecuadas durante su jornada laboral, tales como levantar los brazos por encima de los hombros, extensión y flexión del cuello más allá de los ángulos recomendados, ángulos menores de $90^{\circ}$ en las piernas, entre otras, lo que llega a generar problemas de salud.

El objetivo de este estudio fue evaluar ergonómicamente los miembros superiores y el diseño del área de trabajo de préstamo de libros de una biblioteca en una universidad pública, con la finalidad de conocer el riesgo de trabajo que implica utilizar el mobiliario sin considerar las medidas correctas.

\section{METODOLOGÍA}

En la primera fase se realizó un estudio descriptivo, de corte transversal mediante la observación de la actividad desarrollada por los dos únicos trabajadores (una mujer y un hombre) de 52 años (hombre) y 55 años (mujer). Ambos con una antigüedad de 20 años y 25 años trabajando de tiempo completo (8 horas) en el área de préstamos de la biblioteca.

En la segunda fase se realizó la recolección de datos con el consentimiento de los propios trabajadores, a los cuales se les explico la razón del estudio, la recolección inicio con la grabación en video de la tarea y ciclos de trabajo, así mismo, se tomaron las medidas antropométricas de ambos trabajadores. Con el propósito de evaluar la carga postural y el riesgo músculo esquelético en el módulo de préstamos, se determinó el grado de exigencia física que requiere la actividad y se evaluaron las condiciones fisiológicas, biomecánicas y antropométricas del área de trabajo.

En la tercera fase se procedió a la evaluación ergonómica por medio de los métodos ROSA y REBA. Dichos métodos fueron evaluados con apoyo de las grabaciones de video de cada trabajador, considerando únicamente los movimientos y posturas con vistas en posición lateral y posterior, dichos videos con duración de dos horas para cada posición, grabadas con una cámara Sony HDR-SR11. Para ello, se analizaron las posturas adoptadas por los trabajadores en el área de trabajo, considerando los ciclos de trabajo de préstamos, devoluciones y multas, definidos en la forma SPP-011 de la biblioteca. Posteriormente se analizaron los videos para visualizar en los ciclos de trabajo las tareas que permitan determinar los movimientos y posturas de los trabajadores, con apoyo del software Kinovea - 0.8.15, y la hoja de cálculo REBA (García, 2010) 


\section{RESULTADOS}

\section{Mediciones Antropométricas}

Se realizaron las mediciones antropométricas para ambos trabajadores con base en las mediciones requeridas para cada ciclo de trabajo, lo cual permitió determinar las posturas adecuadas del equipo y del mismo. (Tabla 1)

Tabla 1. Mediciones antropométricas

\begin{tabular}{|l|c|c|}
\hline MEDICIONES EN POSICIÓN SENTADA & $\begin{array}{c}\text { Mujer } \\
(\mathrm{cm})\end{array}$ & Hombre (cm) \\
\hline ALTURA DEL ASIENTO A LOS OJOS & 64,5 & 74 \\
\hline ALTURA DEL ASIENTO AL CODO EN 90 & 19,3 & 20,5 \\
\hline ALTURA AL MUSLO & 51,5 & 50,5 \\
\hline ALTURA DEL CENTRO DEL PUÑO CON LOS BRAZOS HACIA ARRIBA & 101,5 & 104,5 \\
\hline ALTURA DEL SUELO AL HUECO POPLITEO & 38,5 & 40 \\
\hline ALTURA DEL SUELO A LA RODILLA & 45 & 47,5 \\
\hline LONGITUD DEL HUECO POPLITEO AL RESPALDO DE LA SILLA & 42,5 & 45,5 \\
\hline ANCHO DE LA CADERA. SENTADO & 42,5 & 41,5 \\
\hline LARGO DEL BRAZO RESPECTO A LA PARED & 67,5 & 82,5 \\
\hline ALTURA DE LA CABEZA AL SUELO. SENTADO & 115,5 & 118 \\
\hline LONGITUD DE LA RODILLA AL RESPALDO DE LA SILLA & 52 & 55,5 \\
\hline MEDICIONES EN POSICIÓN DE BIPEDESTACIÓN & 160 & 165,5 \\
\hline ALTURA AL CODO & & 94,5 \\
\hline ANCHO DE LOS BRAZOS EXTENDIDOS LATERALMENTE & & 100 \\
\hline
\end{tabular}

\section{Mediciones del Módulo de Trabajo}

Con base en las mediciones antropométricas obtenidas, se procedió a realizar las mediciones del área y se determinó la posición y alcances del equipo del puesto de trabajo. (Figura 1). 


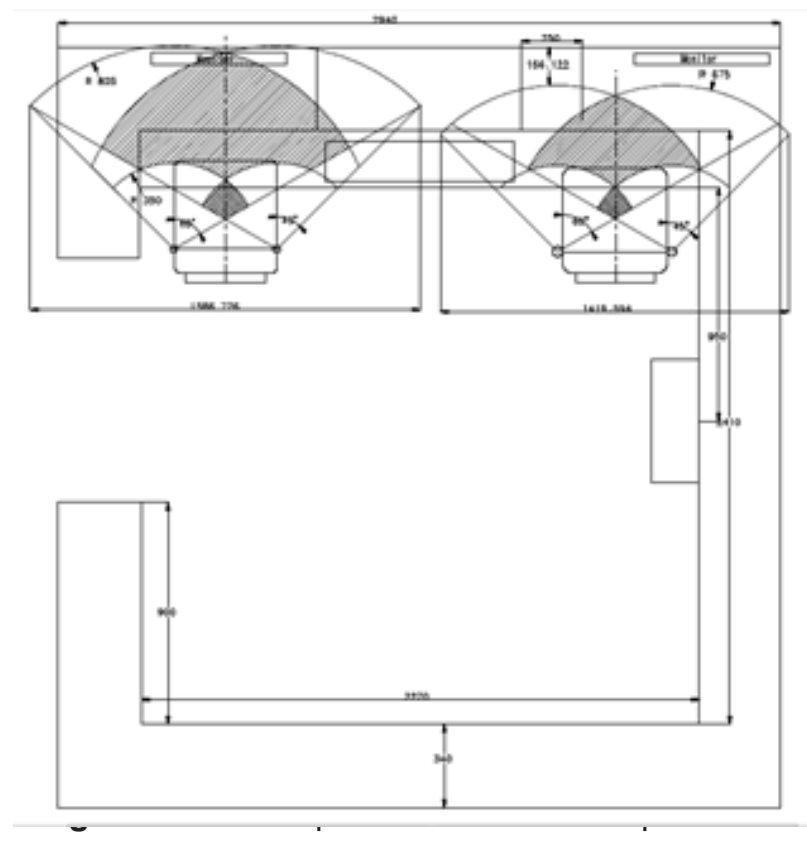

En las Figura 2 y Figura 3 se observa un acercamiento por área, para cada trabajador

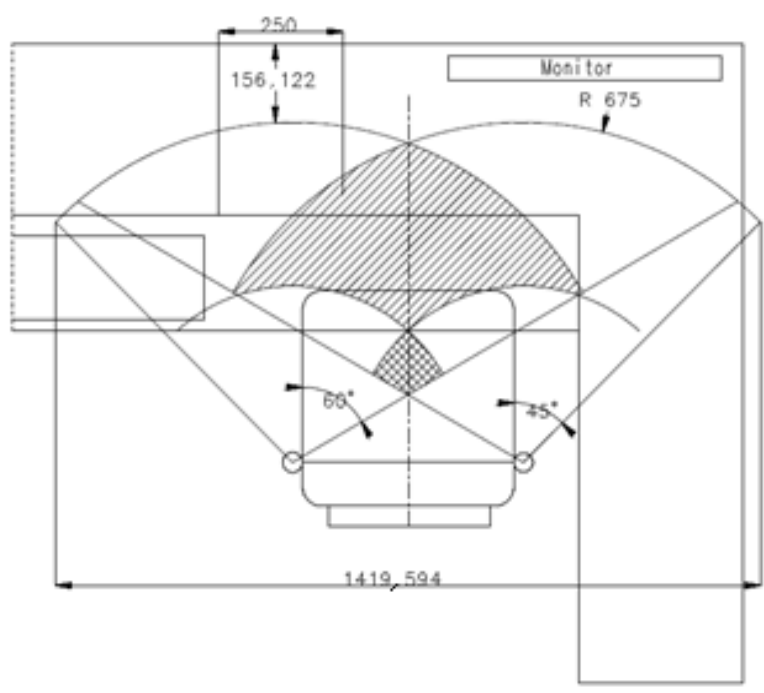

Figura 2. Vista superior del alcance del trabajador mujer. 


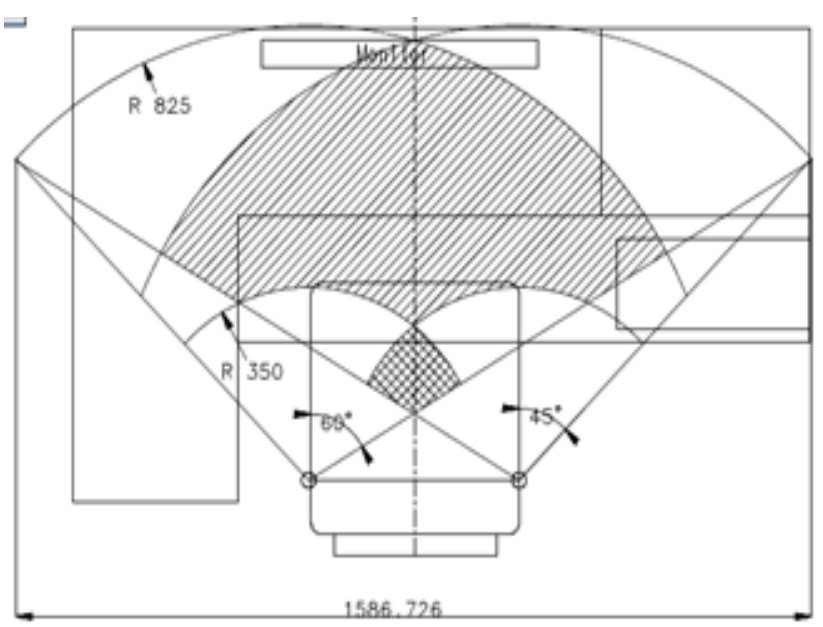

Figura 3. Vista superior del alcance del trabajador hombre.

La metodología de evaluación se muestra en la figura 4. La evaluación ROSA da como resultado un valor de 6 para ambos trabajadores, lo que implica una intervención inmediata para corregir las posturas en ambos trabajadores. Identificando que la postura en la silla y el uso del mouse resultan las más significativas. (Tabla 2). Estos resultados concuerdan con investigación realizada por Lema (2016).

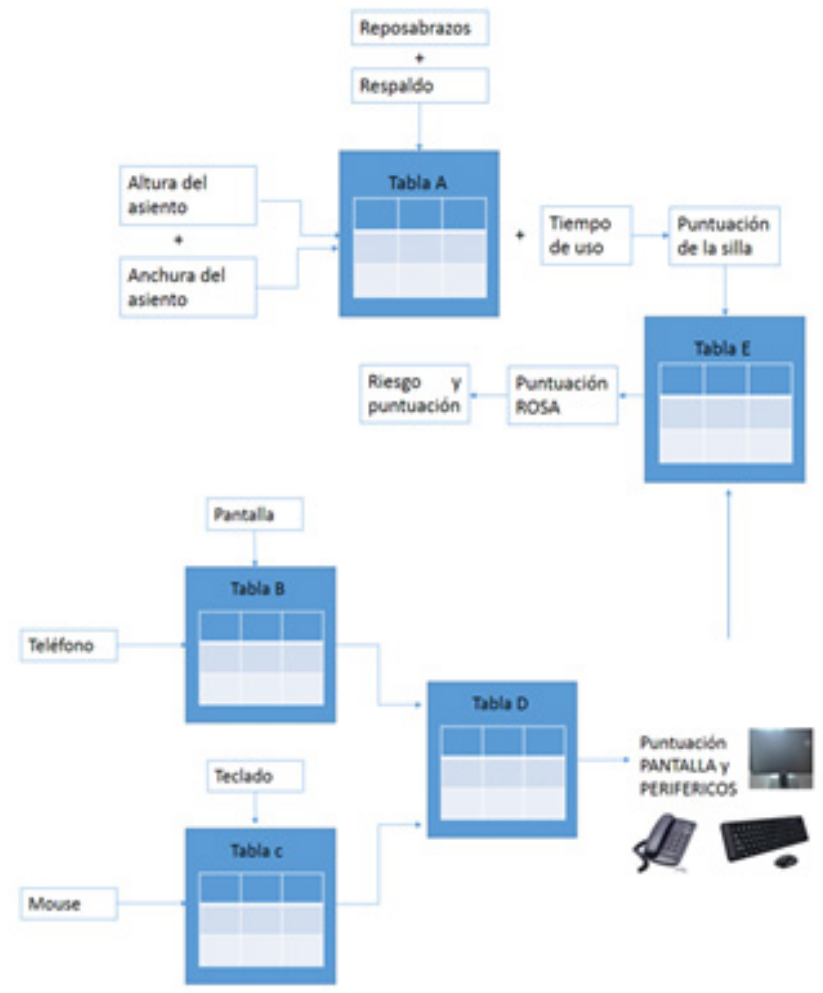

Figura 4. Análisis de puntuación del método ROSA 
Tabla 2. Puntuación ROSA de los trabajadores del módulo de préstamos

\begin{tabular}{|c|c|c|c|c|c|c|}
\hline Trabajador & Sección A & Sección B & Sección C & Sección BC & Sección BC y A & FINAL \\
\hline & 6 & & & & & \\
\hline
\end{tabular}

En cuanto al método REBA, se observa que en la mujer se requiere una actuación cuanto antes, debido al alto nivel de riesgo en su condición postural, en tanto para el hombre, es necesaria una actuación debido al nivel de acción 2. (Tabla 3)

Tabla 3. Puntuación final REBA de los trabajadores del módulo de préstamos

\begin{tabular}{|c|c|c|c|c|}
\hline Trabajador & $\begin{array}{c}\text { Puntuación } \\
\text { final REBA }\end{array}$ & $\begin{array}{c}\text { Nivel de } \\
\text { acción }\end{array}$ & $\begin{array}{c}\text { Nivel de } \\
\text { riesgo }\end{array}$ & Actuación \\
\hline & 9 & 3 & Alto & $\begin{array}{c}\text { Es necesaria } \\
\text { la actuación } \\
\text { cuanto antes }\end{array}$ \\
\hline
\end{tabular}

Para el análisis postural mediante el método REBA se ha dividido en tres ciclos de trabajo: préstamo, devolución y multas, que son los ciclos que más repiten por hora laborada.

En las Figura 5 y Figura 6 se observan las condiciones posturales en las que desarrollan el ciclo de trabajo de préstamo para ambos trabajadores.

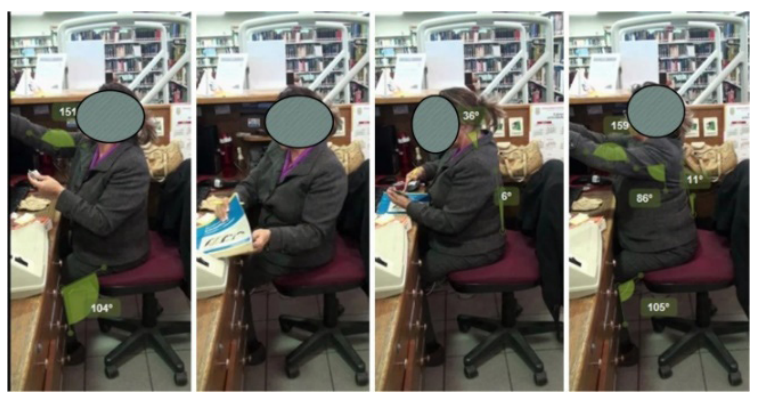

Figura 5. Posturas de la mujer en el ciclo de préstamo 

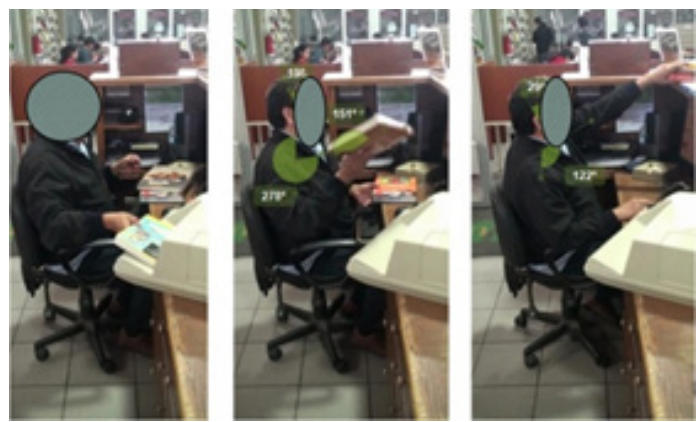

Figura 6. Posturas del hombre en el ciclo de préstamo

En las Figura 7 y Figura 8 se observan las condiciones posturales en las que desarrollan el ciclo de trabajo de devolución los trabajadores. Las Figuras 9 y 10 muestran las condiciones posturales en las que desarrollan el ciclo de trabajo de multas ambos trabajadores.

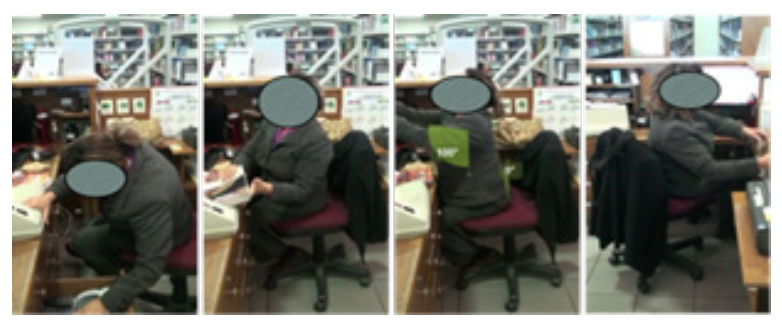

Figura 7. Posturas de la mujer en el ciclo de devolución
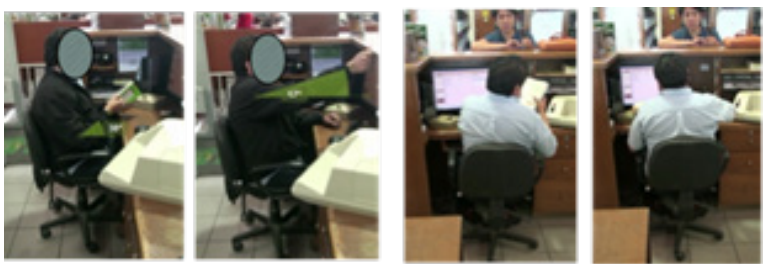

Figura 8. Posturas del hombre en el ciclo de devolución
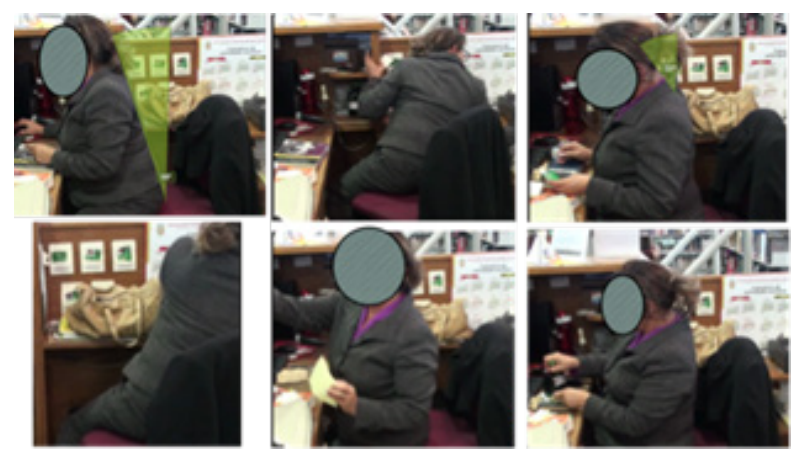

Figura 9. Posturas de la mujer en el ciclo de multas 

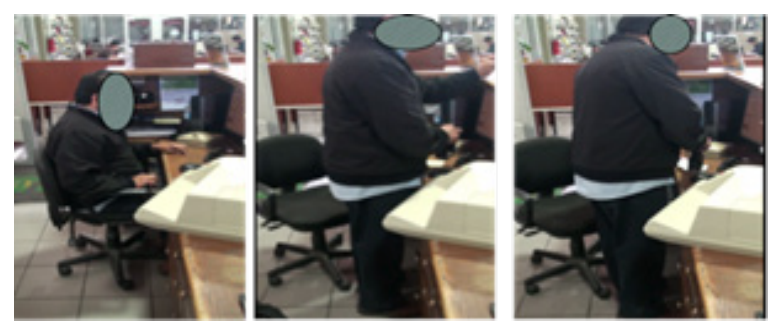

Figura 10. Posturas del hombre en el ciclo de multas

Los resultados de la evaluación REBA, las puntuaciones significativas son para el grupo B específicamente en los brazo para los ciclos de préstamos y devolución, que incluye antebrazos, muñeca, brazos, agarre, y donde los brazos tienen un mayor nivel de riesgo según su puntuación (6) y considerando el resultado por ciclo de trabajo en devolución, la evaluación para ambos trabajadores implica acción inmediata en la tarea (Tabla 4).

Tabla 4. Puntuación REBA de los trabajadores del módulo de préstamos

\begin{tabular}{|c|c|c|c|c|c|c|}
\hline \multirow[b]{3}{*}{ Evaluación REBA } & \multicolumn{2}{|c|}{ Préstamo } & \multicolumn{2}{|c|}{ Devolución } & \multicolumn{2}{|c|}{ Multa } \\
\hline & $M$ & $\mathrm{H}$ & $M$ & $\mathrm{H}$ & $M$ & $\mathrm{H}$ \\
\hline & & batan & matas & 擮 & in & Prostatis \\
\hline \multicolumn{7}{|l|}{ GRUPO A } \\
\hline Cuello & 3 & 2 & 2 & 2 & 2 & 1 \\
\hline Piernas & 1 & 2 & 1 & 1 & 1 & 1 \\
\hline Tronco & 3 & 1 & 3 & 1 & 4 & 1 \\
\hline Carga & 0 & 0 & 0 & 0 & 0 & 0 \\
\hline \multicolumn{7}{|l|}{ GRUPO B } \\
\hline Antebrazos & 2 & 2 & 1 & 2 & 1 & 1 \\
\hline Muñeca & 3 & 3 & 2 & 3 & 2 & 1 \\
\hline Brazos & 6 & 6 & 6 & 6 & 1 & 1 \\
\hline Agarre & 0 & 0 & 0 & 0 & 0 & 0 \\
\hline $\begin{array}{l}\text { PUNTUACION } \\
\text { FINAL }\end{array}$ & 9 & 6 & 9 & 6 & 4 & 1 \\
\hline $\begin{array}{l}\text { NIVEL DE } \\
\text { ACCION }\end{array}$ & 3 & 2 & 3 & 2 & 2 & 0 \\
\hline $\begin{array}{l}\text { NIVEL DE } \\
\text { RIESGO }\end{array}$ & Alto & Medio & Alto & Medio & Medio & Inapreciable \\
\hline ACTUACIÓN & 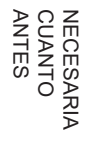 & 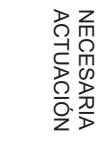 & 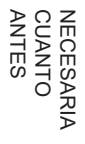 & 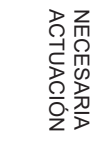 & 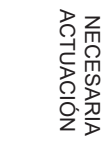 & 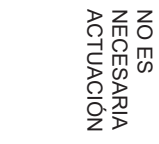 \\
\hline
\end{tabular}


Con base en los resultados, se recomienda implementar modificaciones en el módulo de préstamos de la biblioteca con motivo de mejorar la postura en los trabajadores y disminuir el esfuerzo y movimientos que puedan provocar TME. En la Tabla 5, se visualizan las recomendaciones para atender los resultados obtenidos en el método ROSA, asociados a la postura, monitor, silla, escritorio, descansa pies, teclado, mouse y teléfono, así mismo se mencionan las características a considerar y las medidas deseables para ambos trabajadores. El factor postura, sugiere que la trabajadora mantiene un nivel de riesgo superior por lo que resulta recomendable ajustar la silla, monitor, teclado, mouse y teléfono; en tanto para el trabajador, principalmente se recomienda ajustar el mouse y teclado.

Tabla 5. Recomendaciones con base en el método ROSA

\begin{tabular}{|c|c|c|c|}
\hline Factor & Característica & Medida Mujer & Medida Hombre \\
\hline Postura & $\begin{array}{l}\text { Ángulo entre el torso y el muslo. } \\
\text { Los pies apoyados en el piso y el ángulo del } \\
\text { codo (antebrazo y brazo) }\end{array}$ & $\begin{array}{l}90^{\circ} \\
70^{\circ} \text { y } 120^{\circ}\end{array}$ & $\begin{array}{c}90^{\circ} \\
70^{\circ} \text { y } 120^{\circ}\end{array}$ \\
\hline Monitor & $\begin{array}{l}\text { Colocarse a un brazo de distancia } \\
\text { Entre } 5 \text { y } 8 \mathrm{~cm} \text { debajo de la altura de los ojos }\end{array}$ & $\begin{array}{c}67,5 \mathrm{~cm} \\
56,5-59,5 \mathrm{~cm}\end{array}$ & $\begin{array}{l}82,5 \mathrm{~cm} \\
66-69 \mathrm{~cm}\end{array}$ \\
\hline Silla & $\begin{array}{l}\text { La altura ajustable } \\
\text { El descansabrazos ajustable } \\
\text { Profundidad del asiento } \\
\text { Ancho del asiento }\end{array}$ & $\begin{array}{l}38,5 \mathrm{~cm} \\
19,3 \mathrm{~cm} \\
42,5 \mathrm{~cm} \\
42,5 \mathrm{~cm}\end{array}$ & $\begin{array}{c}40 \mathrm{~cm} \\
20,5 \mathrm{~cm} \\
45,5 \mathrm{~cm} \\
41,5 \mathrm{~cm}\end{array}$ \\
\hline Escritorio & $\begin{array}{l}\text { Altura } \\
\text { Profundidad } \\
\text { Ancho }\end{array}$ & $\begin{array}{l}51,5 \mathrm{~cm} \\
52 \mathrm{~cm} \\
142 \mathrm{~cm}\end{array}$ & $\begin{array}{l}50,5 \mathrm{~cm} \\
55,5 \mathrm{~cm} \\
159 \mathrm{~cm}\end{array}$ \\
\hline Descansa pies & $\begin{array}{l}\text { Profundidad } \\
\text { Ancho } \\
\text { Inclinación }\end{array}$ & $\begin{array}{l}45,7-61 \mathrm{~cm} \\
42,5 \mathrm{~cm} \\
10^{\circ}-25^{\circ}\end{array}$ & $\begin{array}{l}45,7-61 \mathrm{~cm} \\
41,5 \mathrm{~cm} \\
10^{\circ}-25^{\circ}\end{array}$ \\
\hline Teclado & Profundidad (codo a la muñeca) & $38 \mathrm{~cm}$ & $45 \mathrm{~cm}$ \\
\hline Mouse & $\begin{array}{l}\text { Profundidad (codo a la muñeca) } \\
\text { Amplitud de movimiento }\end{array}$ & $\begin{array}{c}38 \mathrm{~cm} \\
35^{\circ}-45^{\circ}\end{array}$ & $\begin{array}{c}45 \mathrm{~cm} \\
35^{\circ}-45^{\circ}\end{array}$ \\
\hline Teléfono & $\begin{array}{l}\text { Profundidad (codo a la muñeca) } \\
\text { Amplitud de movimiento }\end{array}$ & $135^{\circ}-145^{\circ}$ & $135^{\circ}-145^{\circ}$ \\
\hline
\end{tabular}

Con los resultados obtenidos con el método REBA, en la Tabla 6 se sugieren algunas recomendaciones que pudieran dar solución a los niveles de riesgo en las posturas de ambos trabajadores, principalmente en la mujer. Las recomendaciones están vinculadas en atención a las partes con puntuaciones más altas y que son significativas en el resultado final del REBA. 
Tabla 6. Recomendaciones de cambios con base en el método REBA

\begin{tabular}{|c|c|c|c|c|}
\hline Usuario & Ciclo & $\begin{array}{l}\text { Grupo: } \\
\text { parte }\end{array}$ & Recomendación & Características \\
\hline \multirow{2}{*}{ Mujer } & Préstamo & $\begin{array}{l}\text { A: Cuello }=2 \\
\text { B:Brazos }=6\end{array}$ & \multirow{4}{*}{$\begin{array}{l}\text { Espacio para } \\
\text { recepción y en- } \\
\text { trega. }\end{array}$} & \multirow{4}{*}{$\begin{array}{l}\text { Perforación en mueble } \\
\text { de } 48 \text { × } 20 \mathrm{~cm} \text {. }\end{array}$} \\
\hline & Devolución & $\begin{array}{l}\text { A: Cuello }=2 \\
\text { B: Brazos }=6\end{array}$ & & \\
\hline \multirow{2}{*}{ Hombre } & Préstamo & $\begin{array}{l}\text { A: Cuello }=2 \\
\text { B:Brazos }=6\end{array}$ & & \\
\hline & Devolución & $\begin{array}{l}\text { A: Cuello }=1 \\
\text { B: Brazos }=6\end{array}$ & & \\
\hline Mujer & Multas & $\begin{array}{l}\text { A: Cuello }=2 \\
\text { B: Tronco }=4\end{array}$ & $\begin{array}{l}\text { Cambio de } \\
\text { ubicación de la } \\
\text { impresora }\end{array}$ & $\begin{array}{l}\text { Colocar en la parte pos- } \\
\text { terior del área de trabajo } \\
\text { a } 2 \text { metros de distancia, } \\
\text { superficie de } 34 \mathrm{~cm} x \\
44 \mathrm{~cm} \text { a una altura de } \\
123 \mathrm{~cm} \text {. }\end{array}$ \\
\hline
\end{tabular}

En la Figura 11 y Figura 12 se muestra el uso de un espacio que ya existía en el mueble, sin embargo, no se hacía uso de el para la entrega de libros. Con esta investigación, se implementó el uso de este espacio en el mueble, que permita la recepción y entrega de artículos, así como la postura del trabajador al realizar la tarea en los ciclos de préstamo y devolución.

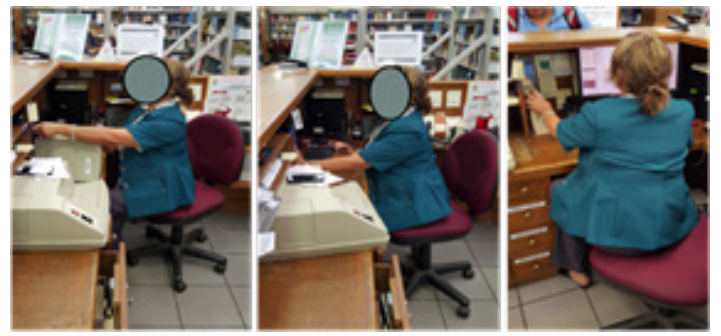

Figura 11. Posturas de la mujer utilizando el espacio de recepción y entrega
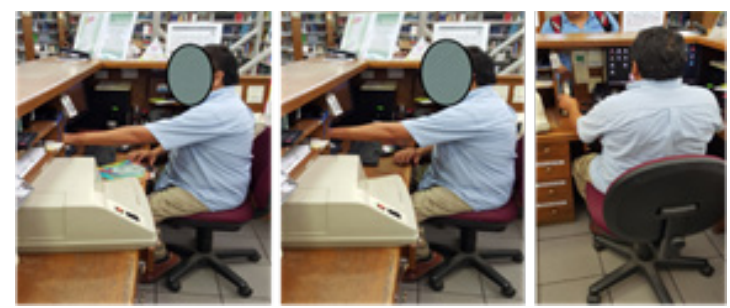

Figura 12. Posturas del hombre utilizando el espacio de recepción y entrega

Al utilizar el espacio, las puntuaciones con respecto a la mujer en el grupo A, análisis del cuello piernas y tronco disminuye significativamente. En el grupo B, el cual comprende antebrazo, 
muñeca, brazos y agarré, también disminuye, resultando más significativa la puntuación de los brazos, lo cual disminuye de nueve a dos puntos, obteniendo una puntuación final en regla de tres con un nivel de acción 1 , nivel de riesgo 1 y puede ser necesaria la actuación.

El las puntuaciones del hombre, usando el espacio también disminuyen, resultando el más significativo en brazos, donde de tener una puntuación, en la actuación final de préstamo y devolución disminuye el nivel de acción, nivel de riesgo bajo de tener un riesgo medio.

Al utilizar el espacio y realizar nuevamente los cálculos para determinar la puntuación del REBA, se obtienen los resultados que se muestran en la Tabla 7, evidenciando una disminución en la puntuación final REBA de 3 para la mujer y 2 para el hombre, ya que se evita el levantamiento de los brazos con flexión $>90^{\circ}$, la flexión o extensión del cuello $>20^{\circ}$, flexión del tronco $>60^{\circ}$ con torsión.

\begin{tabular}{|c|c|c|c|c|c|c|}
\hline \multirow[b]{3}{*}{ EVALUACIÓN REBA } & \multicolumn{2}{|c|}{ PRÉSTAMO } & \multicolumn{2}{|c|}{ DEVOLUCIÓN } & \multicolumn{2}{|c|}{ MULTA } \\
\hline & M & H & M & $\mathrm{H}$ & M & $\mathrm{H}$ \\
\hline & 27 & (5) & mat & IST & & \\
\hline \multicolumn{7}{|l|}{ GRUPO A } \\
\hline CUELLO & 1 & 1 & 1 & 1 & 2 & 1 \\
\hline PIERNAS & 1 & 1 & 1 & 1 & 1 & 1 \\
\hline TRONCO & 1 & 1 & 1 & 1 & 4 & 1 \\
\hline CARGA & 0 & 0 & 0 & 0 & 0 & 0 \\
\hline \multicolumn{7}{|l|}{ GRUPO B } \\
\hline ANTEBRAZOS & 2 & 1 & 2 & 1 & 1 & 1 \\
\hline MUÑECA & 3 & 2 & 3 & 2 & 2 & 1 \\
\hline BRAZOS & 2 & 3 & 2 & 3 & 1 & 1 \\
\hline AGARRE & 0 & 0 & 0 & 0 & 0 & 0 \\
\hline PUNTUACION FINAL & 3 & 2 & 3 & 2 & 4 & 1 \\
\hline NIVEL DE ACCION & 1 & 1 & 1 & 1 & 2 & 0 \\
\hline NIVEL DE RIESGO & $\begin{array}{l}\text { D } \\
\stackrel{\text { D }}{\text { D }}\end{array}$ & $\begin{array}{l}\text { D } \\
\stackrel{D}{D} \\
\text { L }\end{array}$ & 䀡 & $\begin{array}{l}\text { D } \\
\text { ᄅ }\end{array}$ & $\begin{array}{l}\text { 自 } \\
\text { o }\end{array}$ & 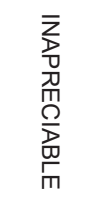 \\
\hline ACTUACIÓN & 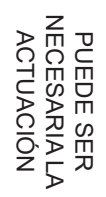 & 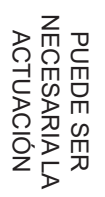 & 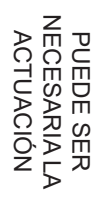 & 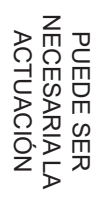 & 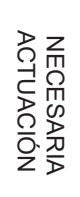 & 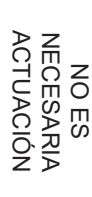 \\
\hline
\end{tabular}

Al disminuir los niveles de actuación y riesgo de ambos trabajadores se considera un avance significativo para disminuir el nivel de riesgo entregar los libros a la altura del brazo por medio 
del espacio en la estación de trabajo, así como implementar de manera permanente el cambio en la ubicación de la impresora. Las anteriores recomendaciones se refieren a cambios físicos del mobiliario, sin embargo, se considera necesario, una campaña de concientización, tanto a los usuarios como a los trabajadores, para un mejor uso de los recursos con los que cuenta la biblioteca y el cuidado de la salud de todos los involucrados en el módulo de préstamo.

En esta evaluación se encontró un mayor riesgo postural en las extremidades superiores del cuerpo al igual que los resultados de Muñoz y Vanegas (2012) en donde, su estudio de oficina demuestra que existe un aumento de carga muscular en cuello y brazos como consecuencia de una postura fija. Resultados concordantes con Nava et al. (2013), en los que se analizaron todas las zonas del cuerpo y en donde los efectos son más significativos en la zona de los hombros.

\section{CONCLUSIONES}

Se evaluó el área de trabajo encontrando en ambos métodos ROSA y REBA resultados que sugerían cambios urgentes a modificar. Una vez que se analizaron las posturas con mayor riesgo se realizaron recomendaciones las cuales fueron atendidas disminuyendo el riesgo.

Las evaluaciones ergonómicas resultan de gran ayuda para mejorar un área de trabajo y reducir la fatiga de los trabajadores, así como las posibilidades de tener TME.

Para este caso, solo un correcto ajuste del mobiliario mejoró el área del trabajo y disminuyo los riesgos de una sobrecarga postural en el trabajo.

Se recomienda ejecutar un programa de reeducación global postural a los trabajadores.

\section{REFERENCIAS}

ROSARIO AMEZQUITA, R.M y AMEZQUITA ROSARIO, TI. (2014). Prevalencia de trastornos músculo-esqueléticos en el personal de esterilización en tres hospitales públicos. Med. segur. trab. [en línea]. 60(234), 24-43. Disponible en: http://dx.doi.org/10.4321/S0465546X2014000100004

ARBELÁEZ, GM., VELÁSQUEZ, SA. y TAMAYO, CM. (2011) Principales patologías osteomusculares relacionadas con el riesgo ergonómico derivado de las actividades laborales administrativas. Revista CES Salud Pública [en línea]. 2(2), 196-203. Disponible en: https:// dialnet.unirioja.es/servlet/articulo?codigo $=3819593$

BOGAERT, I., DE MARTELAER, K., BEUTELS, M., DE RIDDER, K. y ZINZEN, E. (2016). 
Posture analysis among Flemish secondary school teachers: difference between the use of chalkboards and electronic school boards during classroom teaching. Ergonomics [en línea]. 0139(January), 1-7. Disponible en: https://doi.org/10.1080/00140139.2016.1139751.

CASTILLO, JA. y RAMíREZ, BA. (2009) El análisis multifactorial del trabajo estático y repetitivo. Estudio del trabajo en actividades de servicio. Revista Ciencias de la Salud [en línea]. 7(1), 65-82. Disponible en:

DERANGO, K., AMICK, B., ROBERTSON, M., ROONEY, T., MOORE, A. y BAZZANI, L. (2003). The Productivity Consequences of Two Ergonomic Interventions. SSRN [en línea]. 2003(May).Disponible en: https://doi.org/10.2139/ssrn.429180.

DIEGO-MAS, J.A., POVEDA-BAUTISTA, R. y GARZON-LEAL, D.C.(2015). Influences on the use of observational methods by practitioners when identifying risk factors in physical work. Ergonomics [en línea]. 58(10), 1660-1670. Disponible en: https://doi.org/10.1080/00140139. 2015.1023851.

FONSECA, M. y MORAGA, A. (2010). Desórdenes Del Sistema Musculoesquelético Por Trauma Acumulativo En Estudiantes Universitarios De Computación E Informática. Revista de Ciencia y Tecnología, vol. 26, n. 1 y 2, pp. 1-18.

GARCÍA ÁNGEL MARTÍNEZ (2010). Instituto de Seguridad y Salud Laboral de la Región de Murcia.Disponible:http://www.infopreben.com/index.php/riesgos-itsaspreben/ item/364-excel-para-aplicaci\%C3\%B3n-del-m\%C3\%A9todo-reba-de-evaluaci\%C3\%B3nergon\%C3\%B3mica

GENAIDY, A.M., AL-SHEDI, A.A. y KARWOWSKI, W. (1994). Postural stress analysis in industry. Applied Ergonomics [en línea]. 25(2), 77-87. Disponible en: https://doi. org/10.1016/0003-6870(94)90068-X.

HIGNETT, S. y MCATAMNEY, L. (2000). Rapid Entire Body Assessment (REBA). Applied Ergonomics [en línea]. 31(2), 201-205. Disponible en: https://doi.org/10.1016/S00036870(99)00039-3

LASOTA, A.M. (2014). A Reba-Based Analysis of Packers Workload: A Case Study. Scientific Journal of Logistics [en línea].10(1), 87-95. Disponible en: http://www.logforum.net/ pdf/10_1_9_14.pdf

LEMA MEDINA, Á.M. Evaluación de la carga postural y su relación con los trastornos músculo esqueléticos, en trabajadores de oficina de la Cooperativa de Ahorro y Crédito Indígena SAC Ltda. [en línea]. Tesis de Licenciatura. Universidad Técnica de Ambato. Facultad de Ingeniería en Sistemas, Electrónica e Industrial. Carrera de Ingeniería Industrial en procesos de Automatización, 2016. Disponible en: http://repositorio.uta.edu.ec/handle/123456789/24027

LIEBREGTS, J., SONNE, M. y POTVIN, J.R. (2016). Photograph-based ergonomic evaluations using the Rapid Office Strain Assessment (ROSA). Applied Ergonomics [en línea], 52, 317 324. Disponible en: https://doi.org/10.1016/j.apergo.2015.07.028

LÓPEZ TORRES, B.P., GONZÁLEZ MUÑOZ, E.L., COLUNGA RODRÍGUEZ, C. y OLIVA LÓPEZ, E. (2014). Evaluación de Sobrecarga Postural en Trabajadores: Revisión de la Literatura. Ciencia \& trabajo [en línea]. 16(50), 111-115. Disponible en: http://dx.doi. 
MATOS, M. y AREZES, P.M., 2015. Ergonomic Evaluation of Office Workplaces with Rapid Office Strain Assessment (ROSA). Procedia Manufacturing [en línea], 3(2015), 4689-4694. Disponible en: https://doi.org/10.1016/j.promfg.2015.07.562

MINSAL. 2012. Norma Técnica de Identificación y Evaluación de Factores de Riesgo Asociados a Trastornos Musculoesqueléticos Relacionados al Trabajo (TMERT) de Extremidades Superiores. Departamento de Salud Ocupacional [en línea], Chile. Disponible en: http://web. minsal.cl/portal/url/item/cbb583883dbc1e79e040010165014f3c.pdf.

MUÑOZ POBLETE, C.F. y VANEGAS LOPEZ, J.J. (2012). Association between computational jobs and musculoskeletal symptoms in office workers. Medicina y Seguridad del Trabajo [en línea]. 58(227), 98-106. Disponible en: http://dx.doi.org/10.4321/S0465-546X2012000200003

NAVA R., CASTRO J., ROJAS L. y GÓMEZ, M. (2013) Evaluación ergonómica de los puestos de trabajo del área administrativa. Redieluz. vol. 3, n. 1-2, pp: 27-35.

NATARÉN, JANTHÉ JUNO; ELÍO, MARIANO NORIEGA.(2004) Los trastornos musculoesqueléticos y la fatiga como indicadores de deficiencias ergonómicas y en la organización del trabajo. Salud de los Trabajadores, 2004, vol. 12, no 2, p. 27-41.

POULIAKAS, K. y THEODOSSIOU, I. (2013). The economics of health and safety at work: An interdiciplinary review of the theory and policy. Journal of Economic Surveys [en línea]. 27(1), 167-208. Disponible en: https://doi.org/10.1111/j.1467-6419.2011.00699.x.

PRIETO, A., BARRIOS, Y.A. y GONZ, A.S. Aplicación del Método Rosa para la Evaluación de Riesgos Posturales en Oficinas. [en línea]. Universidad de Matanzas, Monografías. 2018. ISBN: 978-959-16-4235-6. Disponible en: http://monografias.umcc.cu/monos/2018/FCE/ mo18206.pdf

ROBERTSON, M.M., CIRIELLO, V.M. y GARABET, A.M. (2013). Office ergonomics training and a sit-stand workstation: Effects on musculoskeletal and visual symptoms and performance of office workers. Applied Ergonomics [en línea], 44(1), 73-85. Disponible en: https://doi. org/10.1016/j.apergo.2012.05.001

REBELO, F.; CARVALHO, R.; BARREIROS, L. Ergonomic Evaluation of Office Chairs. En Proceedings of the Human Factors and Ergonomics Society Annual Meeting.Los Angeles: SAGE Publications, 2000. p. 6-86.

SONNE, M., VILLALTA, D.L. y ANDREWS, D.M. (2012). Development and evaluation of an office ergonomic risk checklist: ROSA - Rapid office strain assessment. Applied Ergonomics [en línea]. 43(1), 98-108. Disponible en: https://doi.org/10.1016/j.apergo.2011.03.008.

WINEMAN, J. (2015). Office Design and Evaluation An Overview. Evironment and behavior [en línea]. 14(3), 271-298. Disponible en: https://doi.org/10.1177/0013916582143002 